\title{
STM of mixed alkylthiol self-assembled monolayers on $\mathrm{Au}(111)$
}

\author{
H. Klein*, N. Battaglini, B. Bellini, Ph. Dumas \\ GPEC UMR CNRS 6631, Case 901, Faculté des Sciences de Luminy, F-13288 Marseille Cedex 9, France
}

\begin{abstract}
Mixed self-assembled monolayers (SAMs) consisting of $n$-alkylthiol and/or 11-mercapto1-undecanol are produced and investigated by Scanning Tunnelling Microscopy (STM) in picoampere regime. Such systems allow the creation of surfaces with tuned chemical properties. We investigate both the qualities of the organic films and their electron transport properties. We evidence a contact regime and measure the decay lengths both in the film and in the tunnelling gap.
\end{abstract}

Keywords: Alkylthiol; Self-assembled monolayers; Scanning Tunnelling Microscopy

\section{Introduction}

Self-assembled monolayers (SAMs) are molecular monolayers chemically bonded to a substrate. They are spontaneously formed during the immersion of a substrate in a solution of surfactant molecules. Their formation can be viewed as a two-step process: chemical bonding of the molecules to the surface, followed by self-assembly favoured by thermal diffusion and van der Waals interactions [1]. The relatively easy chemical preparation of SAMs results in ultra-thin films (typical thickness around $10 \AA$ ). Among the many systems that can lead to SAMs [2,3], $n$-alkylthiols $\left(\mathrm{CH}_{3}-\left(\mathrm{CH}_{2}\right)_{n-1}-\mathrm{SH}\right)$ on gold are the most studied ones. Indeed, $n$-alkylthiol SAMs on Au(111) are the prototype SAM exhibiting a good crystalline quality and a long-term chemical stability. Scanning Probe Microscopy is a first class tool for surface analysis at the nanometric scale and is thus suitable for SAMs studies. In particular, Scanning Tunnelling Microscopy (STM) was successfully applied to organic alkylthiol systems on gold down to molecular resolution [4]. Once formed, the structure of the SAMs is not frozen. It was shown that the exchange between molecules grafted onto the surface and the molecules in solution was possible with $[5,6]$ or without [7] an intentional externally applied force. This feature can be used to chemically functionalize SAMs in order to build new structures with novel properties (e.g. Ref. [8]). These processes lead to mixed SAMs exhibiting domains of two different chemical properties. Moreover, SAMs' 2D crystals can be used as a matrix for the study of the

\footnotetext{
* Corresponding author.

E-mail address: klein@gpec.univ-mrs.fr(H. Klein).
}

properties (e.g. electronic) of isolated specific molecules $[9,10]$. Before such study, it is of prime importance to harvest a good knowledge of the properties of the matrix, and more particularly, its electronic properties. Several studies reported electron tunnelling through isolated molecules [11,12] or through SAMs [13-15].

In this study, we report on the formation and STM observations of mixed SAMs ( $n$-alkylthiols) with $n=8$ or 10 and 11-mercapto1-undecanol), and address some opened questions related to electron transfer through such insulating organic films.

\section{Experimental}

\subsection{SAMs elaboration}

$\mathrm{N}$-alkylthiol SAMs are grafted onto thin Au(111) films evaporated on mica. SAMs elaboration is achieved by immersing fresh gold substrates in $10^{-3} \mathrm{M}$ solutions of alkylthiols in dichloromethane or absolute ethanol for at least $2 \mathrm{~h}$. Samples are then extensively rinsed successively with ethanol and dichloromethane and dried under nitrogen flow prior to STM imaging. The detailed procedure of the sample preparation is described elsewhere [16].

We have prepared mixed SAMs using two methods: either by immersing gold surfaces in solutions with various ratios of the two components, or by immersing an octa- or a decanethiol SAM in millimolar solutions of the other component (11 mercapto1-undecanol for this study). We favour the first method for two components with similar chemical properties and the second one when molecules differ in their chemical properties. 


\subsection{STM}

STM imaging in constant current mode is performed in ambient conditions or in decane. Our experimental setup allows regulation currents down to $0.5 \mathrm{pA}$. STM tips are prepared by cutting $\mathrm{Pt} / \mathrm{Ir}$ (80:20) wires with diameters of $0.25 \mathrm{~mm}$. Due to the strong insulating nature of alkylthiol molecules (saturated carbon chains with a HOMO/LUMO gap around $9 \mathrm{eV}$ [1]), STM imaging on alkylthiol films is only possible with a tunnel resistance in the teraohm range. $I(z)$ data presented in this study was recorded by measuring on STM images the height variation related to a change in the regulation tunnelling current. This procedure, which has to be repeated for each data point of $I(z)$, is relatively lengthy but averages height measurements on image cross-sections leading to good statistics.

\section{STM results}

\subsection{STM images}

Our main goal is the elaboration of mixed SAMs with tuned chemical properties. Since STM is not a chemical probe, we must separate the topographic signature of an isolated molecule from the unavoidable defects of a good quality mono-component SAM, as the one presented in Fig. 1. This image recorded on a decanethiol film shows that the SAM consists of ordered molecular domains separated by defects. In ordered molecular domains, alkyl chains mainly exhibit a compact hexagonal $(\sqrt{3} \times \sqrt{3})$ $R 30^{\circ}$ overlayer of the $\mathrm{Au}(111)(1 \times 1)$ surface, as reported first by Widrig et al. [17]. We measured an intermolecular

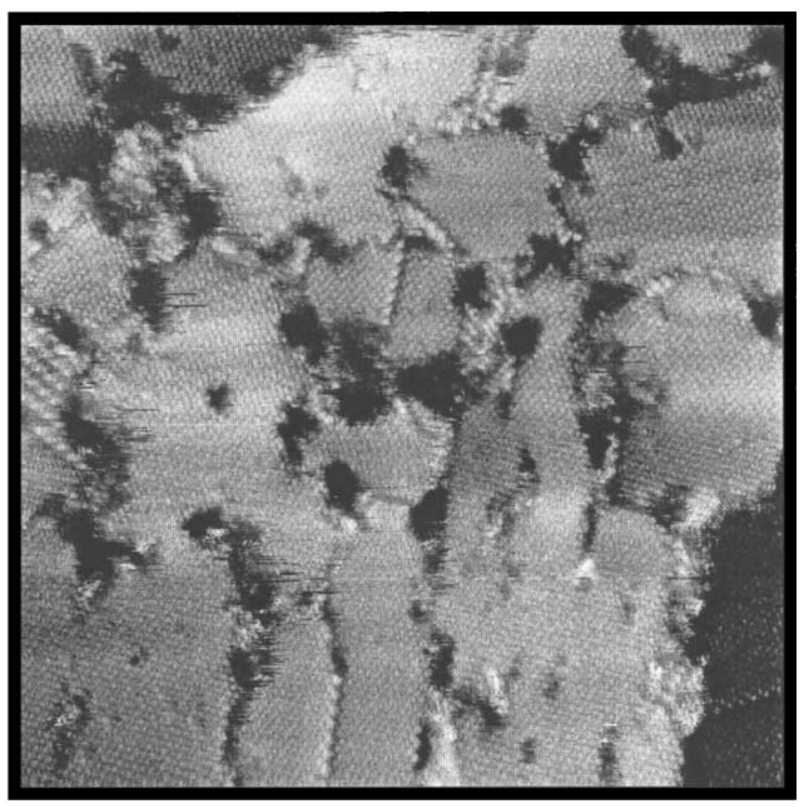

Fig. 1. Typical STM image obtained on a decanethiol SAM on Au(111). Scan size: $51.2 \times 51.2 \mathrm{~nm}^{2}, V_{\mathrm{t}}=-0.9 \mathrm{~V}, I_{\mathrm{t}}=2 \mathrm{pA}$. spacing of $0.5 \mathrm{~nm}$, which is in good agreement with the structural data. The structure of these SAMs observed by STM has been extensively discussed in the literature [18-24].

Following the protocol described in the Experimental section, we have elaborated mixed SAMs consisting of molecules with identical end-groups but different chain lengths, namely $n$-alkylthiols octanethiol $(n=8)$ and decanethiol $(n=10)$. The upper image of Fig. 2 presents the STM image (scan size: $25.6 \times 25.6 \mathrm{~nm}^{2}, z$ scale: $1 \mathrm{~nm}$ ) recorded on the SAM of octane and decanethiol prepared by immersing a gold surface in a solution containing octanethiol/decanethiol (99:1) at a total concentration of 1 $\mathrm{mM}$ in absolute ethanol for $12 \mathrm{~h}$. On this image, we clearly differentiate short and long alkylthiol chains identified as bright spots. The surface is covered by $10 \%$ of the decanethiol, while the ratio in the solution is $1 \%$. This is in agreement with the fact that chains with different lengths and/or different chemical end-groups have different adsorption kinetics $[1,8,25]$, leading to complicated self-assembly mechanisms $[6,26]$.

We measure the height difference between short and long chains of $1.7 \pm 0.2 \AA$ to compare to the known structural height difference of $2.2 \AA$ (assuming that the molecules are in all trans zigzag configuration and are tilted $30^{\circ}$ to the normal of the surface [27]).

The two bottom images of Fig. 2 illustrate that the observed STM contrast is strongly dependent on the tunnelling parameters. We observe a contrast between short and long chains while tunnelling with a current of $2 \mathrm{pA}$ (Fig. 2a). This contrast disappears for a tunnelling current increased up to $10 \mathrm{pA}$ (image b). This effect recorded on the same area of the mixed SAM is reversible and cannot be attributed to a tip change.

Mixed SAMs of decanethiol and 11-mercapto1-undecanol (an alcohol-ended 11-alkylthiol chain) exhibit the same general trends while offering local chemical functionalization. These samples were prepared by immersing freshly prepared decanethiol SAMs in a $1 \mathrm{mM}$ solution of 1-mercapto1-undecanol for $1 \mathrm{~h}$. Fig. 3 presents a typical STM image obtained with a tunnelling current of $2 \mathrm{pA}$. Alcohol termination is interesting from a chemical point of view because such end-group is suitable for the selective grafting of complex chemical groups onto the top of the SAM.

These experiments prove that it is possible to isolate and observe molecules in a matrix, but they pose the question of the origin of the STM contrast on such insulating samples.

\subsection{STM contrast}

A simple assumption about the electron transfer through an organic-insulating SAM would be that electrons are tunnelling directly from one metallic electrode to the other 


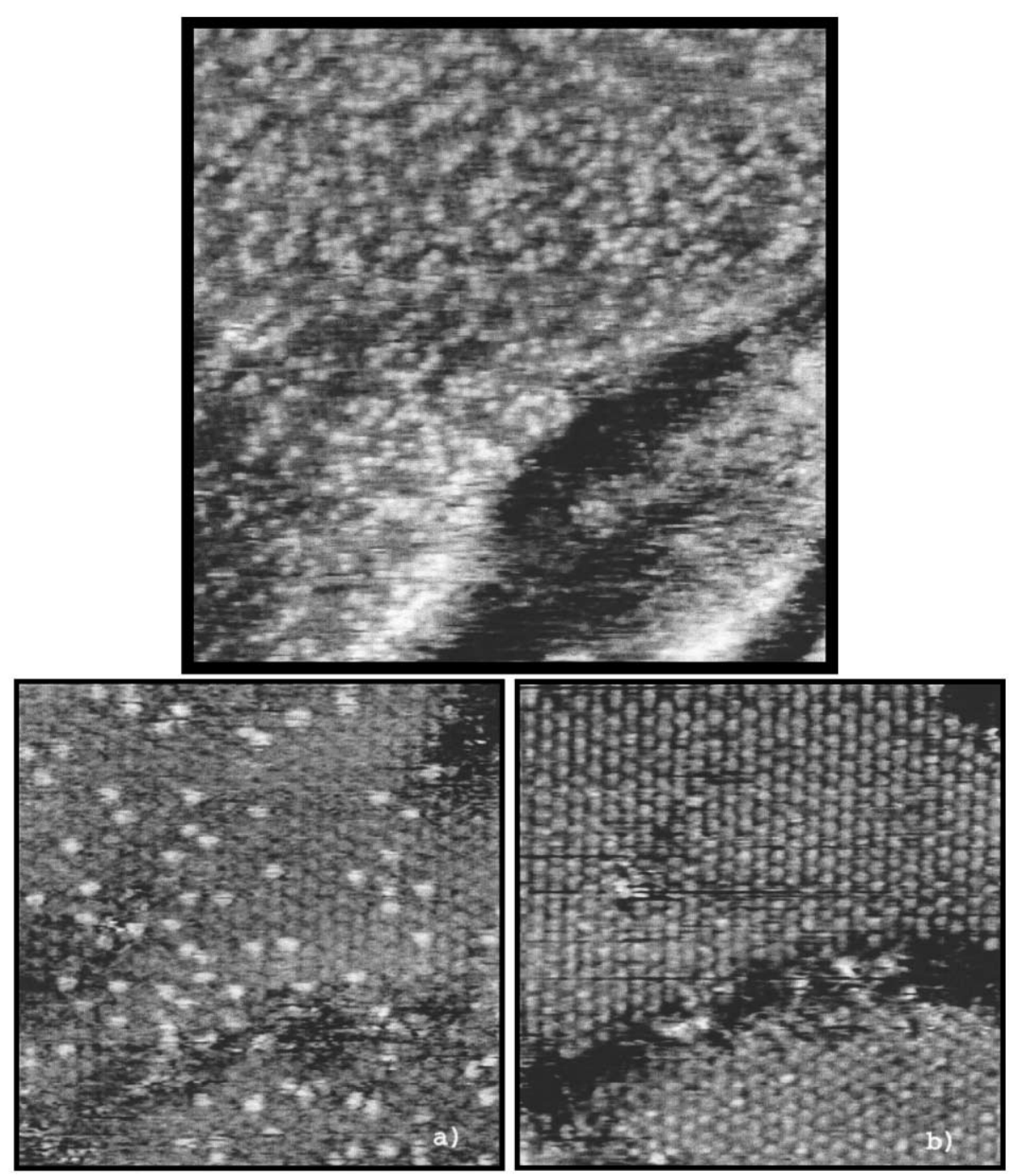

Fig. 2. Top: STM image obtained on a mixed SAM of decanethiol and octanethiol on Au(111). Scan size: $25.6 \times 25.6 \mathrm{~nm}^{2}, V_{\mathrm{t}}=-0.9 \mathrm{~V}, I_{\mathrm{t}}=2 \mathrm{pA}$. The height difference between long and short chains is $1.7 \pm 0.2 \AA$. Bottom: The influence of tunnelling parameters on the observed contrast is clear when considering images (a) and (b) recorded on the same area of the surface. Scan size: $12.8 \times 12.8 \mathrm{~nm}^{2}, V_{\mathrm{t}}=-0.9 \mathrm{~V}, I_{\mathrm{t}}=2 \mathrm{pA}$ for (a) and $10 \mathrm{pA}$ for (b).

one. Within this framework, the alkyl part of the organic chains does not even modulate the electron tunnelling through the gap and does not contribute to the contrast mechanism. The contrast of the STM images would thus be dominated by the HOMO and LUMO orbitals contribution, mainly located on the sulphur atoms. However, this simple assumption is not supported by the STM images presented in Figs. 2 and 3, while we clearly recognise two types of chains. Indeed, we recognise two different lengths ( $n=8,10$ or 11) whatever the chemical end-groups (methyl or alcohol) might be. This shows that the so-called insulating part of the alkylthiol molecules plays a role in the electron transfer from the surface to the tip. Moreover, the experimental height difference between the octane matrix and the isolated decanethiol chains is far from being negligible and was measured to be around $1.7 \AA$. This highlights the contribution of electron transfer through the alkyl part of the chains. This experimentally measured height difference can now be compared to the known structural height difference of $2.2 \AA$ that would be measured for a perfectly conducting layer. To analyse quantitatively these data, following Bumm et al. [28], we apply a model of a tunnel junction described by two layers in series. One layer is the alkylthiol film and the other one is the air or the decane gap. The transconductance $G$ across each layer $i$ is $G_{i}=A_{i} \exp \left(-\alpha_{i} d_{i}\right)$ where $A_{i}$ is the contact conductance, $\alpha_{i}$ is the decay constant, and $d_{i}$ is the thickness of the considered layer. The total transconductance is the product of the individual transconductance: $G_{\text {total }}=G_{\text {film }} G_{\text {gap }}$. In this framework, a simple treatment of a mixed SAM composed of alkyl chains of different lengths leads to:

$\Delta h_{\mathrm{STM}}=\Delta h_{\text {film }}\left(1-\alpha_{\text {film }} / \alpha_{\text {gap }}\right)$

where $\Delta h_{\mathrm{STM}}$ is the experimentally measured height difference between long and short chains, and $\Delta h_{\text {film }}$ is the 
known structural height difference. The analysis of the data allows the determination of the ratio of the decay lengths $\alpha_{\text {film }} / \alpha_{\text {gap }}=0.23$. This model provides an explanation of the observed contrast and contributes to our understanding of the electron transfer through the organic layer. As shown hereafter, $I(z)$ spectroscopy is a complementary way towards the knowledge of the electron transfer.

\subsection{I(z) measurements on decanethiol SAM}

Experiments were conducted in decane, a highly insulating liquid, to prevent instabilities due to the changes in the composition of the tunnelling gap. To avoid difficulties related to the interruption of the regulation loop, $I(z)$ data were recorded as explained in the Experimental section. Keeping the tip bias equal to $-900 \mathrm{mV}$ and the regulation loop always active, sequences $I_{0}(2 \mathrm{pA}) \rightarrow I \rightarrow I_{0}$ are repeated for different values of $I$ (between 1 and $40 \mathrm{pA}$ ). For each value $I$, we measure the associated height variation $\Delta_{z}(I)$. Data are then represented by plotting $I\left(\Delta_{z}\right)$. Fig. 4 shows two typical sets of data measured with two different tips on a decanethiol SAM. The reproducibility of the curves demonstrates that the effects are insensitive to the tip shape. The $I(z)$ spectroscopy exhibits an exponential decrease of $I$ with $\Delta_{z}$ at a large distance and a singularity around $I \simeq 10 \mathrm{pA}$. The large distance behaviour is due to the well-known exponential dependence of the tunnel current with the inter-electrode distance. We assign the observed singularity to the point contact when the tip skims the top of the organic layer. A similar behavior has recently been reported by Salmeron et al. [14]

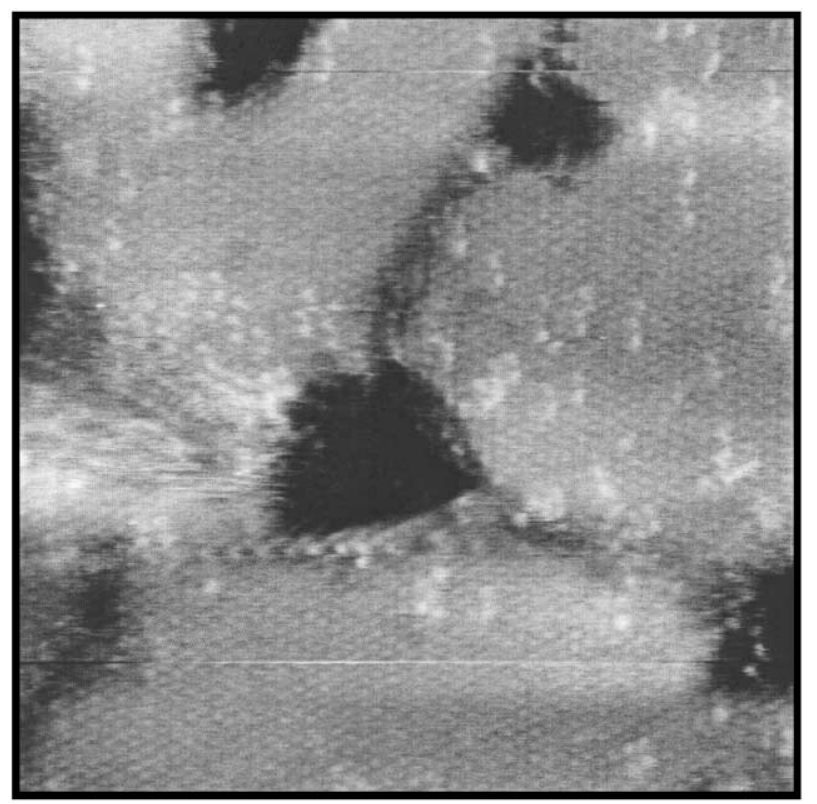

Fig. 3. STM image obtained on a mixed SAM decanethiol and mercapto undecanol on $\mathrm{Au}(111)$. Scan size: $25.6 \times 25.6 \mathrm{~nm}^{2}, V_{\mathrm{t}}=-0.9 \mathrm{~V}, I_{\mathrm{t}}=2$ pA.

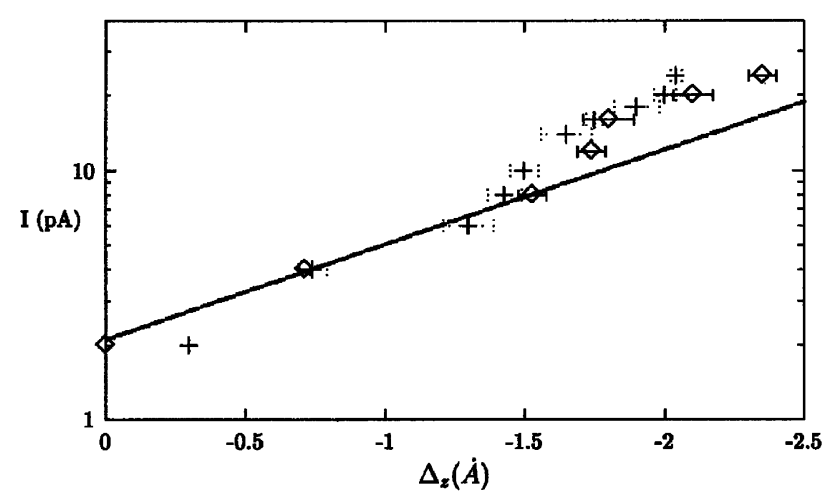

Fig. 4. $I\left(\Delta_{z}\right)$ measurements performed in decane on decanethiol SAM with two different tips. The $I\left(\Delta_{z}\right)$ spectroscopy exhibits an exponential decay of $I$ with $\Delta_{z}$ at large distances and a singularity around $I \simeq 10 \mathrm{pA}$. The observed singularity corresponds to the point contact when the tip skims the top of the organic layer. The solid line is a plot of $f(x)=$ $A \exp \left(-\alpha \Delta_{z}\right)$ for $\alpha=0.97$.

on conjugated thiols. Though the tip interacts with the organic layer for the tunnelling current above $10 \mathrm{pA}$, this interaction does not damage the surface, and molecular resolution images can still be obtained for currents up to $50 \mathrm{pA}$. The analysis of the evolution of the images while increasing the regulation current in this interacting regime is beyond the scope of this communication and will be discussed elsewhere. True non-contact tunnelling is thus only attainable for tunnel impedance greater than $100 \mathrm{G} \Omega$. The determination of the point contact allows the evaluation of the altitude at which the tip flies over the organic layer. For a decanethiol layer and our typical operating conditions $(I=2 \mathrm{pA}$ and $V=-900 \mathrm{mV})$, this value is $1.5 \pm 0.5 \AA$. Though the number of points is limited, from the exponential decay of the $I\left(\Delta_{z}\right)$ curve, we estimate the decay constant of the tunnelling gap: $\alpha_{\text {gap }} \simeq 1 \AA^{-1}$. Then, from Eq. (1), we calculate the decay constant of the organic film $\alpha_{\text {film }} \simeq 0.2 \AA^{-1}$. These experimental values are in discrepancy with the calculated values reported in Ref. [28], which also forecasts a contact point but for 10 times lower tunnelling impedances. Further experimental evidences of this behavior are needed and are currently investigated to reach a deeper understanding of electron transport through organic molecules.

\section{Conclusion}

We have produced and imaged mixed SAMs with an STM in the picoampere regime. These mixed SAMs consist of $n$-alkythiols $(n=8,10)$ and 11-mercapto1-undecanol. The observed STM contrast of mixed SAMs is explained by the model with two tunnel barriers in series. I( z) spectroscopic data evidence a transition to a contact regime for currents higher than $10 \mathrm{pA}$. The determination of the absolute tip/sample distance leads to a value of 1.5 $\AA$ lower than published theoretical expectations. This work 
contributes to an improved knowledge of the electron transport through organic films at the nanometric scale.

\section{References}

[1] A. Ulman, An Introduction to Ultrathin Organic Films, Academic Press, Boston, 1990.

[2] A. Ulman, MRS Bull. 6 (1995) 46-51.

[3] A. Ulman, Chem. Rev. 96 (1996) 1533-1554.

[4] G.E. Poirier, Chem. Rev. 97 (1997) 1117-1127.

[5] G. Liu, M.B. Salmeron, Langmuir 10 (1994) 367-370.

[6] S. Xu, S.J.N. Cruchon, J.C. Gamo, G.Y. Liu, J. Chem. Phys. 108 (1998) 5002-5012.

[7] J.B. Schlenoff, M. Li, H. Ly, J. Am. Chem. Soc. 117 (1995) $12528-12536$.

[8] J.P. Folkers, P.E. Laibinis, G.M. Whitesides, Langmuir 8 (1992) 1330-1341.

[9] M.T. Cygan, T.D. Dunbar, J.J. Arnold, L.A. Bumm, N.F. Shedlock, T.P. Burgin, L. Jones II, D.L. Allara, J.M. Tour, P.S. Weiss, J. Am. Chem. Soc. 120 (1998) 2721-2732.

[10] J. Chen, M.A. Reed, C.L. Asplund, A.M. Cassell, M.L. Myrick, A.M. Rawlett, J.M. Tour, P.G. Van Patten, Appl. Phys. Lett. 75 (5) (1999) 624.

[11] V.J. Langlais, R.R. Schlitter, H. Tang, A. Gourdon, C. Joachim, J.K. Gimzewski, Phys. Rev. Lett. 83 (14) (1999) 2809-2812.

[12] B.C. Stipe, M.A. Rezaei, W. Ho, Science 280 (1998) 1732.
[13] N. Camillone III, C.E.D. Chidsey, G. Liu, G. y Scoles, J. Phys. Chem. 98 (1993) 3503

[14] M. Salmeron, G. Neubauer, A. Folch, M. Tomitori, D.F. Ogletree, P. Sautet, Langmuir 9 (1993) 3600-3611.

[15] T. Ishida, W. Mizutani, N. Choi, U. Akiba, M. Fujihira, H. Tokumoto, J. Phys. Chem. B 104 (2000) 11680-11688.

[16] H. Klein, W. Blanc, R. Pierrisnard, C. Fauquet, Ph. Dumas, Eur. Phys. J. B 14 (2000) 371-376.

[17] C.A. Widrig, C.A. Alves, M.D. Porter, J. Am. Chem. Soc. 113 (1991) 2805.

[18] G. Nelles, H. Schönherr, M. Jaschke, H. Wolf, M. Schaub, J. Küther, W. Tremel, E. Bamberg, H. Ringsdorf, H.J. Butt, Langmuir 14 (1998) 808-815.

[19] S. Chiang, Science 272 (1996) 1123.

[20] C. Schönenberger, J. Jorritsma, J.A.M. Sondag-Huethorst, L.G.J. Fokkink, J. Phys. Chem. 99 (1995) 3259-3271.

[21] G.E. Poirier, M.J. Tarlov, Langmuir 10 (1994) 2853-2856.

[22] L.G.J. Fokkink, C. Schönenberger, J.A.M. Sondag-Huesthorst, J. Jorristsma, Langmuir 10 (1994) 611-614.

[23] D. Anselmetti, A. Baratoff, H.J. Güntherhodt, E. Delamarche, B. Michel, Ch. Gerber, H. Kang, H. Wolf, Europhys. Lett. 27 (5) (1994) 365-370.

[24] G.E. Poirier, E.D. Pylant, Science 272 (1996) 1145-1148.

[25] K. Tamada, M. Hara, H. Sasabe, W. Knoll, Langmuir 13 (1997) $1558-1566$.

[26] R. Yamada, K. Uosaki, Langmuir 14 (1998) 855-861.

[27] E. Barrena, C. Ocal, M. Salmeron, J. Chem. Phys. 113 (6) (2000) $1-6$.

[28] L.A. Bumm, J.J. Arnold, T.D. Dunbar, D.L. Allara, P.S. Weiss, J. Phys. Chem. B 103 (1999) 8122-8127. 